\title{
Effect of Surface Waves on Air-Sea Momentum Exchange. Part II: Behavior of Drag Coefficient under Tropical Cyclones
}

\author{
IL-Ju MoOn, IsAaC Ginis, And Tetsu HaRA \\ Graduate School of Oceanography, University of Rhode Island, Narragansett, Rhode Island
}

(Manuscript received 29 August 2003, in final form 10 April 2004)

\begin{abstract}
Present parameterizations of air-sea momentum flux at high wind speed, including hurricane wind forcing, are based on extrapolation from field measurements in much weaker wind regimes. They predict monotonic increase of drag coefficient $\left(C_{d}\right)$ with wind speed. Under hurricane wind forcing, the present numerical experiments using a coupled ocean wave and wave boundary layer model show that $C_{d}$ at extreme wind speeds strongly depends on the wave field. Higher, longer, and more developed waves in the right-front quadrant of the storm produce higher sea drag; lower, shorter, and younger waves in the rear-left quadrant produce lower sea drag. Hurricane intensity, translation speed, as well as the asymmetry of wind forcing are major factors that determine the spatial distribution of $C_{d}$. At high winds above $30 \mathrm{~m} \mathrm{~s}^{-1}$, the present model predicts a significant reduction of $C_{d}$ and an overall tendency to level off and even decrease with wind speed. This tendency is consistent with recent observational, experimental, and theoretical results at very high wind speeds.
\end{abstract}

\section{Introduction}

The air-sea momentum fluxes (or wind stress) over ocean are key boundary parameters for atmospheric, oceanic, and wave models. In severe storm situations such as tropical cyclones, accurate estimation of the fluxes is crucial in predicting storm track and intensity, storm surges, and ocean wave fields (Ooyama 1969; WAMDI Group 1988; Emanuel 1995; Doyle 2002; Moon et al. 2003b). It has long been recognized that the wind stress (or the drag coefficient $C_{d}$ or the roughness length $z_{0}$ ) depends on wind speed, as well as on the sea state and atmospheric stability (Monin and Obukhov 1954; Charnock 1955; Large and Pond 1981; Smith et al. 1992). However, the bulk formula that is widely used in many numerical models assumes that the wind stress magnitude is a unique function of the wind speed, being independent of the sea state. The roughness length is expressed in terms of the air friction velocity $u_{*}$ and the acceleration of gravity $g$ based on the dimensional argument by Charnock (1955). The bulk parameterizations are mostly based on field measurements in weak-to-moderate wind regimes less than $25 \mathrm{~m} \mathrm{~s}^{-1}$ and predict monotonic increase of the drag coefficient with wind speed.

A number of observational studies indicate that $C_{d}$ strongly depends on the sea state (Toba et al. 1990; Smith et al. 1992; Johnson et al. 1998; Drennan et al.

Corresponding author address: Il-Ju Moon, Graduate School of Oceanography, University of Rhode Island, Narragansett, RI 02882. E-mail: mij@gso.uri.edu
2003). In most cases, the sea state has been represented by the dimensionless wave age $\left(c_{p} / u_{*}\right)$, where $c_{p}$ is the phase speed at the peak frequency. However, the exact dependency of $C_{d}$ (or nondimensional roughness, i.e., Charnock coefficient) on the wave age still remains controversial, especially in high wind and very young sea conditions (Komen et al. 1988; Jones and Toba 2001).

In severe storm conditions, especially in tropical cyclones, the surface wave field is quite complex and fast varying in space and time. Dominant waves often propagate at large and even conflicting angles to the local wind (Moon et al. 2003a). The misaligned waves may influence both the magnitude of the wind stress and the angle between the wind stress and the mean wind (Smith 1980; Geernaert 1988; Rieder et al. 1994; Bourassa et al. 1999; Grachev et al. 2003). For complex seas, therefore, the wind stress must be treated as a vector quantity, and its proper estimation requires using two-dimensional wave spectrum, not a single parameter like the wave age.

There have been a number of studies that attempted to predict air-sea momentum flux (in particular, roughness length) by explicitly calculating the wave-induced stress (i.e., the stress supported by surface waves) with the two-dimensional wave spectrum (Janssen 1989; Chalikov and Makin 1991; Makin et al. 1995; Makin and Mastenbroek 1996; Makin and Kudryavtsev 1999; Hara and Belcher 2004). Among them, Janssen's (1989) theory is most widely used in surface wave models (WAMDI Group 1988) and coupled models between atmosphere (or ocean) and surface waves (Weber et al. 
TABLE 1. Experimental designs.

\begin{tabular}{|c|c|c|c|c|}
\hline Expt name & Storm type & $\operatorname{HTS}\left(\mathrm{m} \mathrm{s}^{-1}\right)$ & $\begin{array}{l}\text { Max wind speed } \\
\text { (intensity) }\left(\mathrm{m} \mathrm{s}^{-1}\right)\end{array}$ & Structure \\
\hline Expt A & $\begin{array}{l}\text { Stationary medium } \\
\text { Typical speed medium } \\
\text { Fast-moving medium }\end{array}$ & $\begin{array}{r}0 \\
5 \\
10\end{array}$ & 45 & Symmetric \\
\hline Expt B & $\begin{array}{l}\text { Fast-moving weak } \\
\text { Fast-moving strong }\end{array}$ & 10 & $\begin{array}{l}35 \\
55\end{array}$ & Symmetric \\
\hline Expt $\mathrm{C}$ & $\begin{array}{l}\text { Asymmetric typical speed medium } \\
\text { Asymmetric fast-moving medium }\end{array}$ & $\begin{array}{r}5 \\
10\end{array}$ & 45 & Asymmetric \\
\hline Expt D & Hurricane Bonnie & Varying & 45 & Asymmetric \\
\hline
\end{tabular}

1993; Lionello et al. 1998; Johnson et al. 1999; Bao et al. 2000; Desjardins et al. 2000; Doyle 2002; Moon 2004). However, no attempts have been made to estimate the wind stress as a vector quantity and to apply it over the extreme wind speeds above $35 \mathrm{~m} \mathrm{~s}^{-1}$.

In the companion paper (Moon et al. 2004, hereafter Part I), we investigated the effect of surface waves on air-sea momentum exchange for mature and growing seas forced by steady uniform winds. Here, the complete wave spectrum was constructed by merging the WAVEWATCH-III (WW3) spectrum in the vicinity of the spectral peak with the spectral tail parameterization based on the equilibrium spectrum model of Hara and Belcher (2002). The result was then incorporated into the wave boundary layer model of Hara and Belcher (2004) to explicitly calculate the wave-induced stress vector, the mean wind profile, and the drag coefficient over any given complex seas. This study follows the approach of Part I but focuses on investigating the effect of surface waves on air-sea momentum exchange in extreme complex seas forced by tropical cyclones. In particular, behavior of drag coefficient (or nondimensional roughness) depending on a hurricane's characteristics is examined. For this purpose, various idealized hurricanes with different translation speeds, intensities, and structures are applied in numerical experiments, and the results are compared with other stress formulas currently in use.

A brief outline of the experimental design and method used to investigate drag behaviors under hurricane forcing is introduced in section 2. Section 3 describes results of the experiments, and they are compared with available observational data. The summary and conclusion are given in section 4 .

\section{Experimental designs}

The present experiments are designed to investigate the effects of the storm translation speed, intensity, structure, and varying storm speed on the stress behavior under hurricane wind forcing (Table 1). First, three idealized hurricanes with different translation speeds are used to examine the effect of the hurricane translation speed (HTS). In these experiments, the maximum wind speed (MWS) is fixed at $45 \mathrm{~m} \mathrm{~s}^{-1}$, and spatially symmetric wind forcing is applied. Second, the influence of the storm intensity is investigated by two fast-moving symmetric hurricanes with different MWSs. The effect of storm structure is investigated by two asymmetric hurricanes moving with the HTS of $5 \mathrm{~m} \mathrm{~s}^{-1}$ (typical speed) and $10 \mathrm{~m} \mathrm{~s}^{-1}$ (fast moving). Finally, to examine the effect of a time-varying translation speed, the observed translation speed of Hurricane Bonnie (1998) is used for the experiment.

The wind fields for these experiments are obtained from an analytical model proposed by Holland (1980). The model requires the centered and ambient pressure and the radius of maximum wind speed (RMW) as inputs. In these experiments, we fix the RMW as $74 \mathrm{~km}$ and ambient pressure as $1012 \mathrm{hPa}$, but centered pressures vary depending on the storm intensity. Figure 1 shows a wind field of a symmetric hurricane estimated by the Holland model. The wind fields are moved northward with various HTSs over the deep ocean of 2000-m depth, extending $3000 \mathrm{~km}$ in the south-north direction and 1500 $\mathrm{km}$ in the east-west direction. The model domain, resolution, and grid system of the WW3 used for these experiments are identical to those used in Part I.

In the following discussions, the results of all numerical experiments except experiment D are presented after a spinup time of $54 \mathrm{~h}$, when a quasi steady state is achieved. The experiment D using Hurricane Bonnie's translation speed is designed for a more realistic simulation of the wind stress and therefore allows an analysis of time-varying translation speeds.

\section{Results and discussion}

\section{a. Effect of storm translation speed on wind stress}

\section{1) Surface WAVE FIELDS}

Figures $2 \mathrm{a}-\mathrm{c}$ represent spatial distributions of the significant wave height $H_{s}$ (contours), and the dominant wave direction and wavelength $L$ (arrows) produced by idealized tropical storms (experiment A) with HTSs of 0,5 , and $10 \mathrm{~m} \mathrm{~s}^{-1}$, respectively. The figures are plotted for locations within a distance of $300 \mathrm{~km}$ from the storm center. The arrow length is proportional to the dominant 


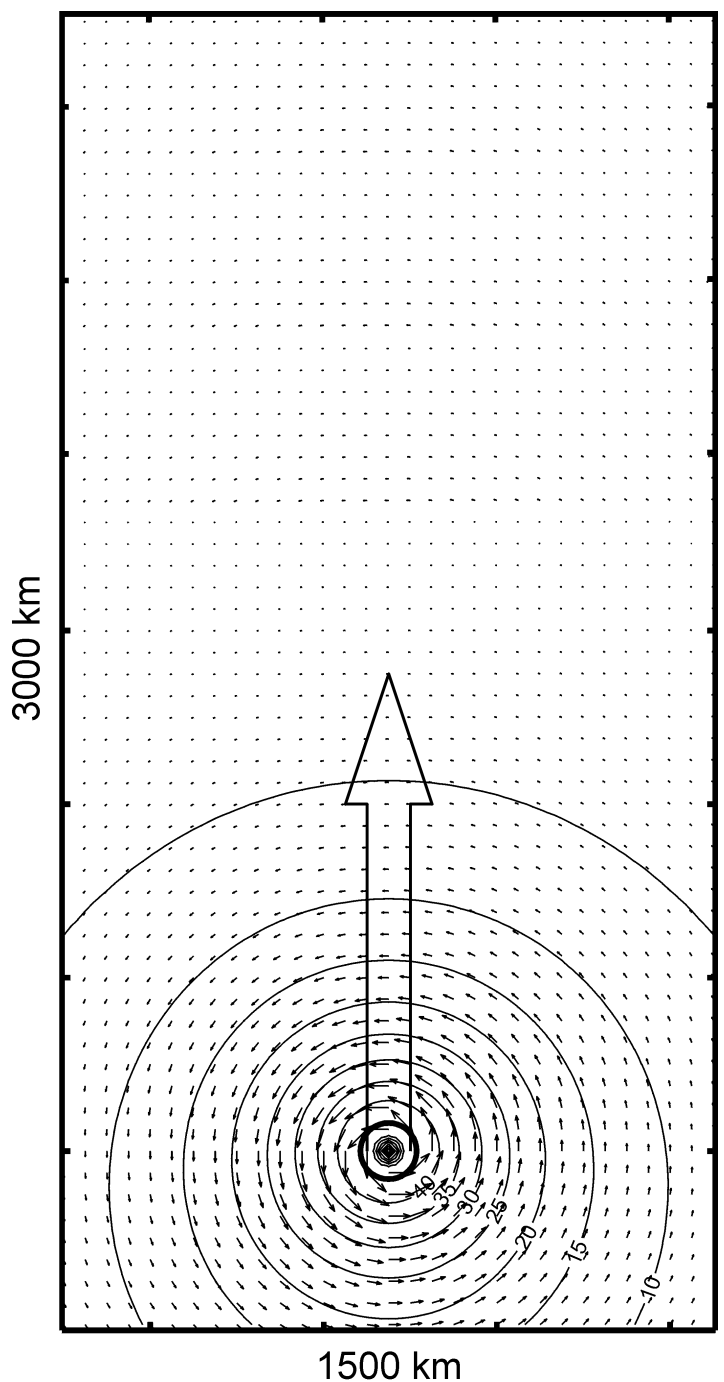

FIG. 1. Wind fields for the idealized experiment, which are moving with various HTSs. These are obtained from an analytic model proposed by Holland (1980). Center pressure ( $953 \mathrm{hPa}$ ) and RMW (74 $\mathrm{km})$ are used as inputs of the model. The thick solid circle represents the radius of maximum wind speed.

wavelength, and a dashed circle is the RMW. As the HTS increases, waves in the front-right quadrant of the storm track become higher and longer, while those in the rear-left quadrant become lower and shorter. The maximum values of $H_{s}$ and $L$ in the fast-moving hurricane reach up to 23 and $420 \mathrm{~m}$, respectively, which are almost twice the values observed in the stationary hurricane.

In this study, the "input" wave age $\left(c_{\mathrm{pi}} / u_{*}\right)$ is determined from the peak input frequency $f_{\mathrm{pi}}$, which is defined as the peak frequency of the wind sea (waves directly forced by wind) part of the spectrum. It is different from the conventional peak frequency $f_{p}$ calculated from the one-dimensional spectrum. With a hurricane-generated complex multimodal wave field, it is essential to find the peak frequency of the wind sea in order to establish a reliable value of the wave age. Estimation of the $f_{\mathrm{pi}}$ is made within the WW3 (Tolman and Chalikov 1996). Distributions of the input wave age representing the state of growth of wind waves relative to local wind forcing are shown in Figs. 2d-f. As the HTS increases it becomes comparable to the group speed of dominant waves. In particular, waves to the front right of the hurricane's track are exposed to prolonged forcing from wind, that is, become "trapped" within the hurricane. The trapped waves produce higher, longer, and older waves in the front-right quadrant. On the other hand, waves to the rear left of the track experience limited fetch and duration of wind forcing. These waves become lower, shorter, and younger as the HTS increases.

Moon et al. (2003a) have concluded that the hurricane-generated wave field is mostly determined by two factors: the distance from the hurricane center and the HTS. They show that for a hurricane with a low translation speed, the wave field is everywhere dominated by waves generated in the vicinity of the RMW, whose direction then depends on the distance from the hurricane center. For a fast-moving hurricane, the dominant waves are mostly determined by resonance (or trapped waves). When the group velocity of dominant waves is close to the HTS, their growth may be significantly enhanced by the resonance effect, and the waves produced by the resonance may dominate the wave system over its propagating areas.

Figure 3 shows the directional wave spectra obtained at selected points. For the stationary hurricane, the spectra have the same shapes in all directions and the dominant waves are locally generated and roughly aligned with the wind. As the storm moves faster, the wave spectra in the east and north points of the hurricane show a unimodal shape due to a resonance effect, while the spectra in the west and south of the hurricane display a more complex structure with multiple peaks.

\section{2) Directional CHARACTERISTICS OF WIND, WIND STRESS, AND WAVES}

Under the influence of hurricanes, the misalignment of winds and waves is common, as seen in Fig. 3, because of the curvature of hurricane-generated wind fields and their translation. Dominant waves generated near the RMW frequently propagate at a large angle to the local wind, sometimes even opposing it. In all existing wind stress parameterizations it is assumed that the surface wind and wind stress vectors are collinear. However, if there are dominant waves propagating at a large angle to the local wind, the surface wind and stress vectors may not be aligned (Smith 1980; Geernaert 1988; Bourassa et al. 1999; Grachev et al. 2003). In this section, we investigate the directional characteristics of wind, wind stress, and waves.

Figure 4 compares directions of wind, dominant waves, and wind stress, which are produced by the ide- 

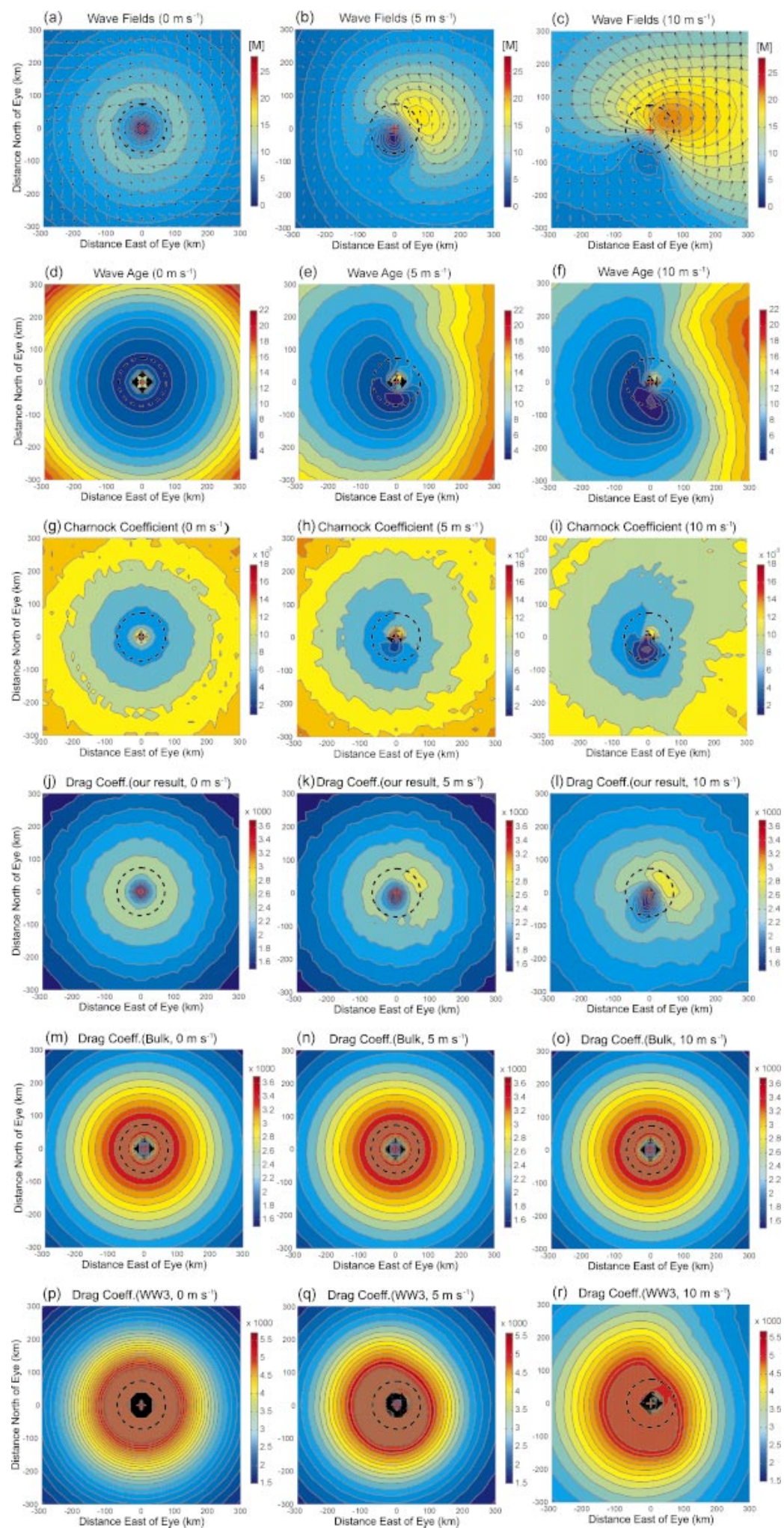

FIG. 2. Spatial distributions of (a)-(c) significant wave height and mean wave direction, (d)-(f) input wave age, (g)-(i) Charnock coefficient $z_{\mathrm{ch}}$, (j)-(l) drag coefficient $C_{d}$ by the present approach, (m)-(o) $C_{d}$ by the bulk formula with $z_{\text {ch }}$ of 0.0185 , and (p) $-(\mathrm{r}) C_{d}$ by the internal prediction of the WW3. All figures are produced by idealized tropical storms (MWS $=45 \mathrm{~m} \mathrm{~s}^{-1}$ ) with an HTS of (left) 0 , (center) 5 , and (right) $10 \mathrm{~m} \mathrm{~s}^{-1}$. The dashed circle and plus symbol represent the RMW and the center of storm, respectively. 

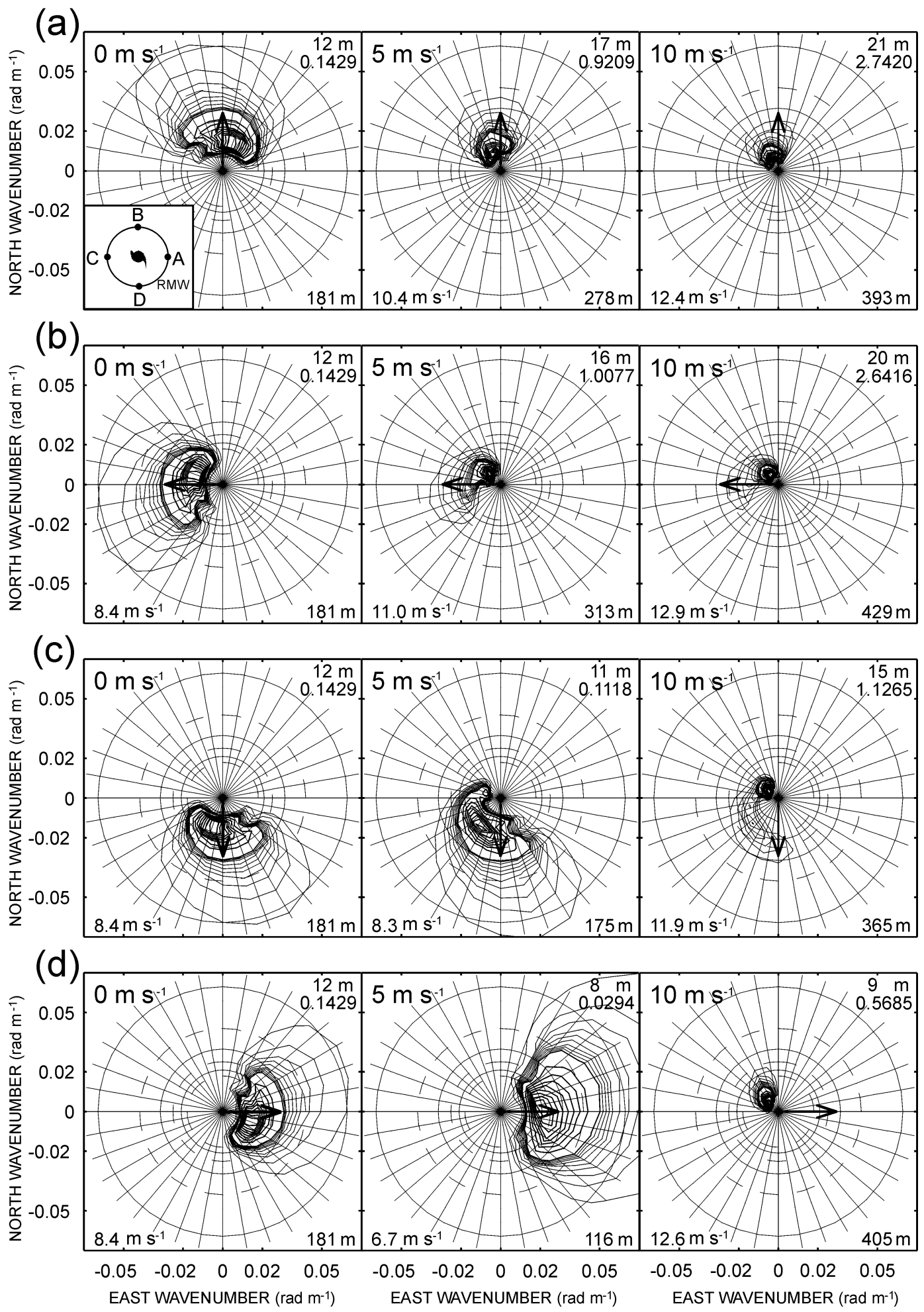

FIG. 3. Directional wave spectra in the WW3 produced by idealized tropical storms with an HTS of (left) 0, (center) 5, and (right) $10 \mathrm{~m} \mathrm{~s}^{-1}$ at (a) east, (b) north, (c) west, and (d) south points located at the distance of RMW from the storm center. The dashed circles (outer to inner) correspond to wavelengths of 150,250 , and $350 \mathrm{~m}$. The solid circles indicate wavelengths of 100,200 , and $300 \mathrm{~m}$. Each spectrum contains 18 contours, linearly spaced from $10 \%$ to $90 \%$ (thick contours) and from $1 \%$ to $9 \%$ (thin contours) of the peak spectral density, which is shown in the second number in the upper-right corner. The thick arrows extend in the downwind direction with their length proportional to the wind speed. A wind speed of $30 \mathrm{~m} \mathrm{~s}^{-1}$ corresponds to a length of $0.03 \mathrm{rad} \mathrm{m}^{-1}$. The total $H_{s}$ is shown in the upper-right corner. The mean wavenumber and group velocity are in the lower-right corner and in the lower-left corner, respectively. 
(a) Vectors of wave, wind, and stress $\left(0 \mathrm{~m} \mathrm{~s}^{-1}\right)$

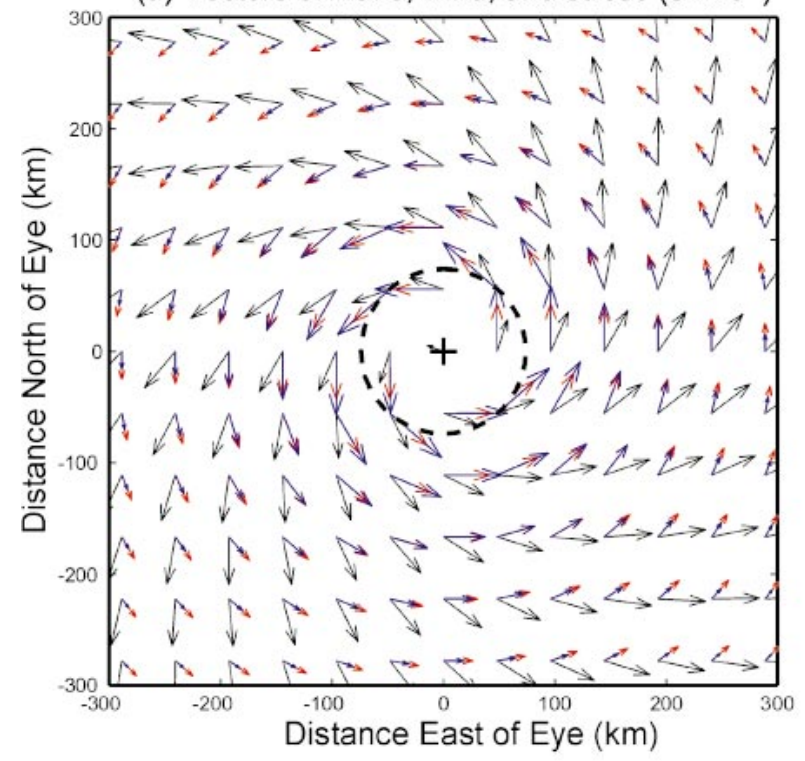

(c) Vectors of wave, wind, and stress $\left(10 \mathrm{~m} \mathrm{~s}^{-1}\right)$

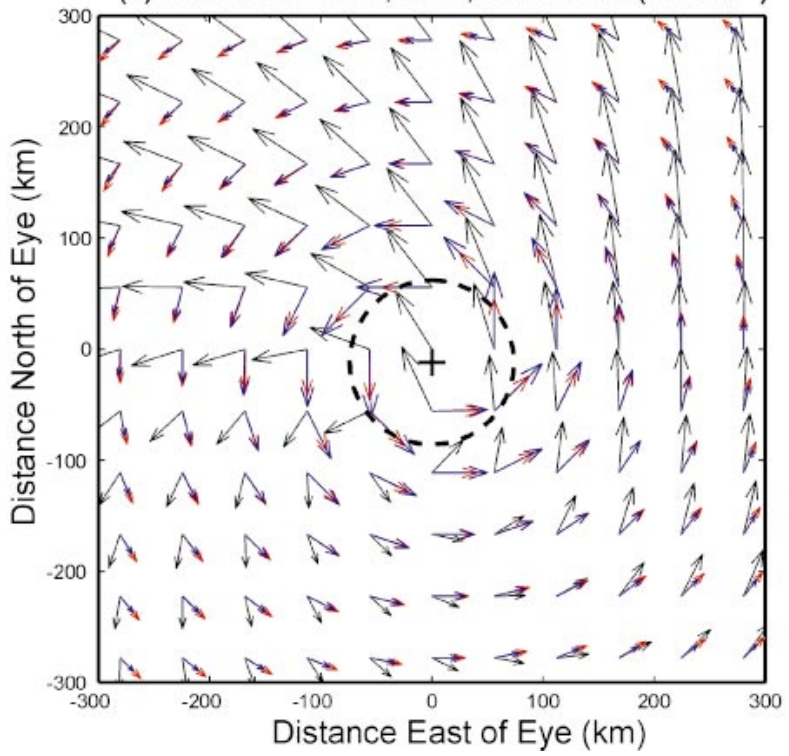

alized hurricanes with different translation speeds. For the stationary hurricane (Fig. 4a), the dominant waves are mainly generated from near the RMW and consistently propagate to the right of the local wind direction. As the HTS increases, the dominant waves are mostly determined by the waves produced by resonance effect, propagating in the hurricane track direction or to the left of the track (Moon et al. 2003a). The maximum directional difference between dominant waves and wind is found in the front-left quadrant of the hurricane, especially in the fast-moving case (Fig. 4c). At these locations, the misalignment between the wind and wind stress is also the largest (the wind stress vectors always (b) Vectors of wave, wind, and stress $\left(5 \mathrm{~m} \mathrm{~s}^{-1}\right)$
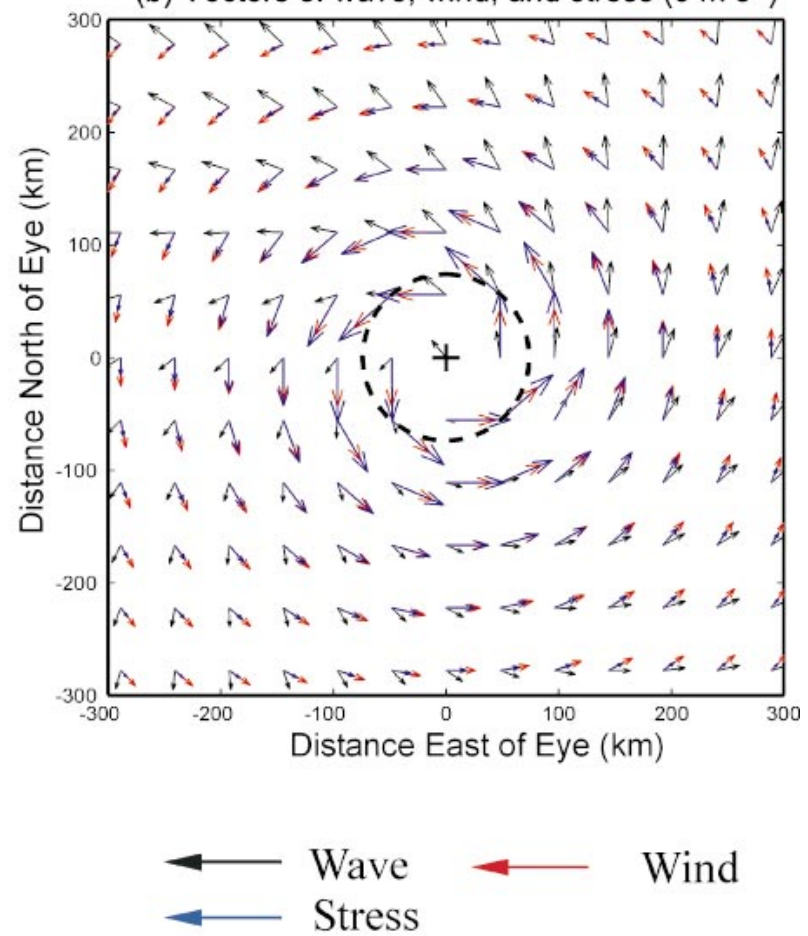

FIG. 4. Comparison of directions among wind (red arrows), dominant wave (black arrows), and wind stress (blue arrows), which are produced by idealized tropical storms (MWS $=45 \mathrm{~m} \mathrm{~s}^{-1}$ ) with translation speeds of (a) 0 , (b) 5 , and (c) $10 \mathrm{~m} \mathrm{~s}^{-1}$. A dashed circle represents the RMW, and a plus symbol represents the center of storm.

lie between the mean wind direction and dominant wave direction), but it never exceeds a few degrees. This is mainly because dominant waves that are misaligned with wind support little momentum flux; that is, the misaligned dominant waves may reduce the wind stress magnitude (compared to the stress with aligned dominant waves), but they cannot affect the stress direction appreciably. Our results show that the WW3 spectrum at the cutoff frequency is always aligned with the wind direction even if the peak region is not aligned. This implies that the equilibrium range that supports the majority of the stress is always aligned with the wind. However, note that this result does not imply that the 
two-dimensional (vector) stress estimation is unnecessary. If the wind and waves are assumed to be always aligned and the stress is treated as a scalar quantity, the stress magnitude may be significantly overestimated.

Observations of Geernaert (1988), Rieder et al. (1994), and Grachev et al. (2003) showed more distinct angle differences between wind stress and mean wind velocity vectors than our results. This may be because their observations were made with a much weaker wind speed and a well-developed swell. In such conditions, the effect of (misaligned) swell (negative momentum flux from waves to wind) may be more enhanced relative to the effect of (aligned) wind seas. The present approach only considers the positive flux and neglects negative flux from swell mainly because we are interested in strong wind regime forced by tropical cyclones. If we include the negative fluxes in this model, the misalignment might be more significant at large distances from the hurricane center where strong misaligned waves and very weak wind forcing exist.

Since the wind and wind stress directions are mostly aligned (with misalignment of less than a few degrees), it is possible to calculate the drag coefficient $C_{d}$, the roughness length $z_{0}$, and the Charnock coefficient $z_{\mathrm{ch}}=$ $g z_{0} / u_{*}^{2}$ from the magnitude of the $10-\mathrm{m}$ wind speed and the wind friction velocity. Such results are discussed next.

\section{3) CHARNOCK COEFFICIENT AND DRAG COEFFICIENT}

Figures $2 \mathrm{~g}-\mathrm{i}$ show the spatial distribution of the Charnock coefficient $z_{\mathrm{ch}}$ estimated from the idealized tropical storm experiments with different translation speeds. For all cases, $z_{\mathrm{ch}}$ is less than 0.018 , which is the value used in the National Oceanic and Atmospheric Administration/Geophysical Fluid Dynamics Laboratory (NOAA/ GFDL) operational hurricane model, within $300 \mathrm{~km}$ from the storm center. As the translation speed increases, a significant decrease of $z_{\mathrm{ch}}$ is found in the rear-left quadrant of the track where younger waves exist.

Figure 5 shows the scatterplot of $z_{\mathrm{ch}}$ as a function of the input wave ages at all grid points of the WW3 simulation (except where the estimated $f_{c}$ is outside the WW3-resolving range). The results are color coded according to the local wind speed at each grid point. The figure clearly shows that most of the decreased $z_{\text {ch }}$ values are found in very young seas (input wave age less than 10) and with strong winds (higher than $30 \mathrm{~m} \mathrm{~s}^{-1}$ ). For a fast-moving hurricane (Fig. 5c), the drop of $z_{\mathrm{ch}}$ with decreasing input wave age is further enhanced. Here, thin solid lines represent the results of numerical experiments with growing seas (Part I) under steady uniform winds at four different wind speeds. Our results under the hurricane wind forcing is roughly consistent with the pure growing sea results; that is, the Charnock coefficient is mainly determined by the two parame- ters-the input wave age and the local wind speedregardless of the complexity of the wave field.

Figures $2 \mathrm{j}-1$ show spatial distributions of $C_{d}$ for three different translation speeds. In the stationary hurricane the maximum of $C_{d}$ appears near the RMW. As the HTS increases, the maximum moves to the front-right quadrant of the track.

In Fig. 6, $C_{d}$ is plotted against wind speed at all grid points as in Fig. 5, with different colors assigned according to the relative position from the storm center. The figure shows that $C_{d}$ in the front-right quadrant monotonically increases with the wind speed (but not as fast as the mature sea results of Part I denoted by the dash-dot lines), while that in the rear-left quadrant tends to level off or even decrease at higher winds for the fast-moving hurricane. The latter trend is similar to the very young sea results (wind duration of $1 \mathrm{~h}$ ) of Part I under steady uniform wind, denoted by the solid lines. This is because in extremely high wind conditions, the equilibrium range of very young waves in the rearleft quadrant is quite low and makes a relatively smaller contribution to the total wind stress than more developed waves in the front-right quadrant.

Next we compare the obtained drag coefficient distributions (Figs. 2j-1) with those of a bulk formula based on a constant $z_{\mathrm{ch}}(0.0185)$ (Figs. 2m-o) and of the internal prediction of the WW3 (Figs. 2p-r). The distributions from the bulk formula show that $C_{d}$ is independent of the HTS because it is independent of the sea state. The $C_{d}$ values from the WW3 are estimated parametrically with their dependence on the input wave age (Tolman 2002); that is, younger waves yield higher drag. The distributions of $C_{d}$ from the WW3 (Figs. 2p-r) are consistent with those of wave age (Figs. 2d-f) as expected. In Fig. 6, our drag coefficient values are compared with the bulk formula (dashed line) and the WW3 estimates. It is seen that the bulk formula and the WW3 significantly overestimates the drag coefficient compared to our results, particularly at higher wind speeds and for faster-moving hurricanes.

In many oceanic and atmospheric models, the bulk formula based on the constant Charnock coefficient (mostly 0.0185) is widely used even with high winds (Kurihara et al. 1998; Johnson et al. 1998). This parameterization is based on extrapolations from field measurements obtained under moderate winds; it has not been validated against observations at high winds. The extrapolation yields the monotonic increase of $C_{d}$ with wind speed (Fig. 6). Our result at high winds above 30 $\mathrm{m} \mathrm{s}^{-1}$, on the other hand, predicts an overall tendency for $C_{d}$ to level off or even decrease with wind speed. Therefore, they suggest that the choice of $z_{\mathrm{ch}}=0.0185$ is a good approximation only in the moderate winds; at high wind speeds the bulk formula may lead to significant overestimation of the momentum exchange across the air-sea interface. 
Wave Age vs. Charnock Coefficient

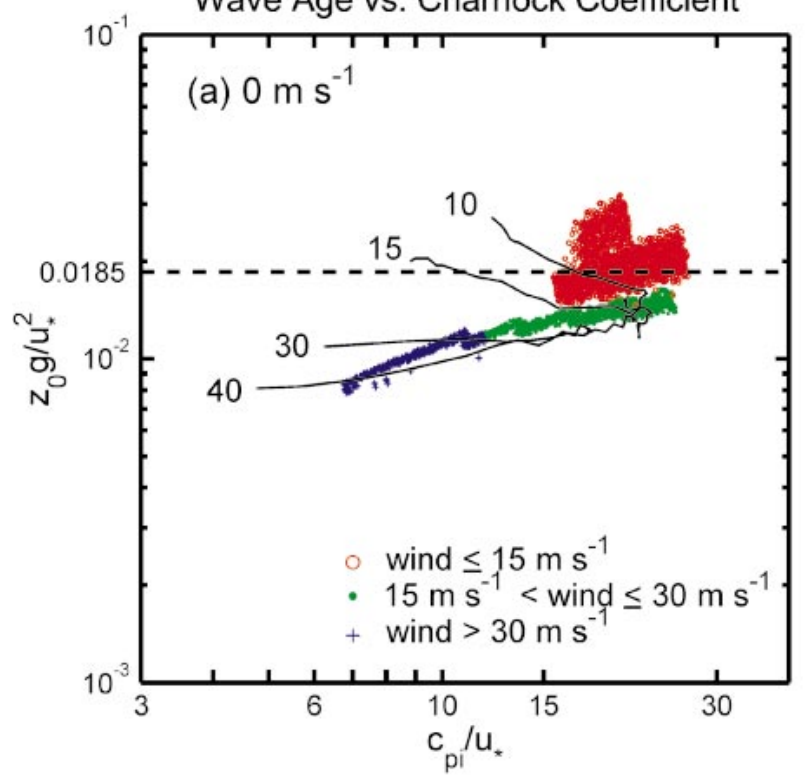

Wave Age vs. Charnock Coefficient

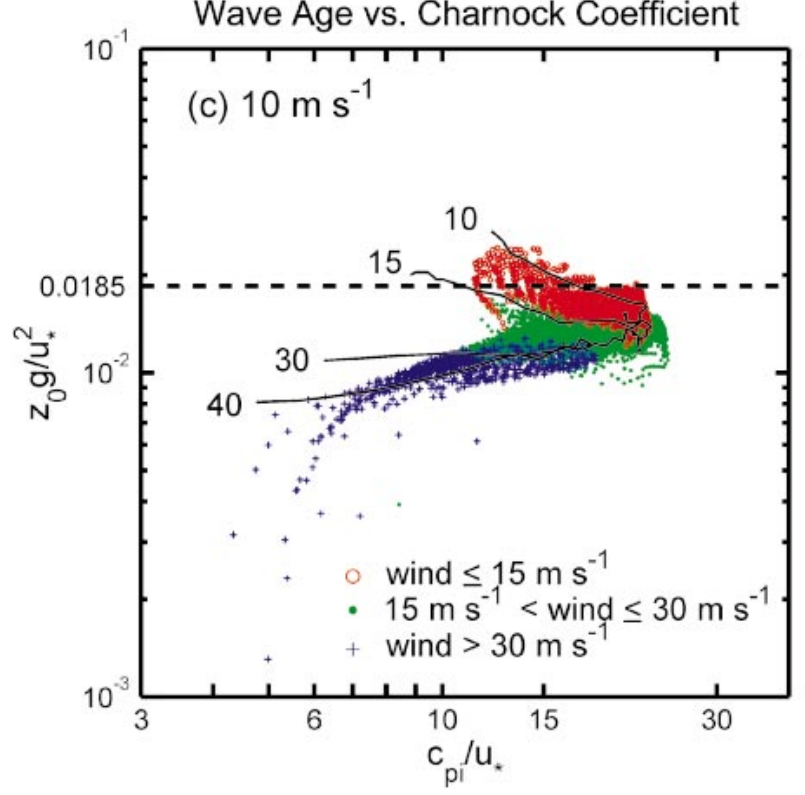

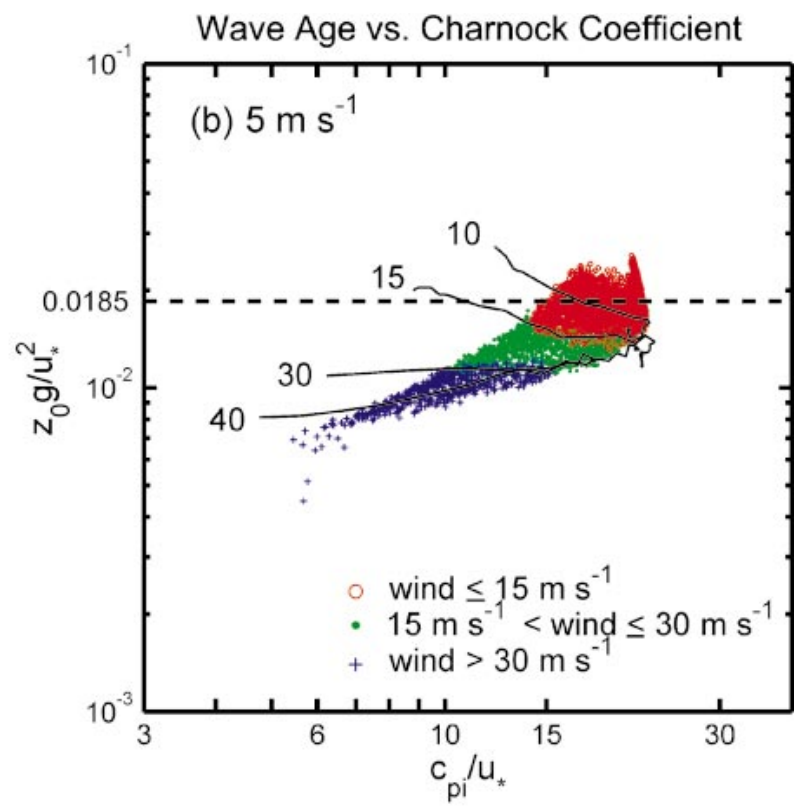

FIG. 5. Scatterplots of the $z_{\mathrm{ch}}$ as a function of wave ages in all grid points for storms with translation speeds of (a) 0 , (b) 5 , and (c) 10 $\mathrm{m} \mathrm{s}^{-1}$. Moderate winds (open circle), medium-speed winds (filled circle), and strong winds (plus) are plotted using different symbols and colors. A dashed line represents the $z_{\mathrm{ch}}$ of 0.0185 . Solid lines are results of Part I obtained using steady uniform winds of 10, 15, 30, and $40 \mathrm{~m} \mathrm{~s}^{-1}$ speeds.

\section{b. Effect of storm intensity}

We next examine how the wind field and the drag coefficient vary with different storm intensity. Typical hurricanes produce the MWS in the range from 30 to $60 \mathrm{~m} \mathrm{~s}^{-1}$. In this section, we investigate the effect of the storm intensity on wind stress using two fast-moving $\left(\right.$ HTS $=10 \mathrm{~m} \mathrm{~s}^{-1}$ ) hurricanes (experiment B) with different intensities, that is, MWSs of 35 (weak storm) and $55 \mathrm{~m} \mathrm{~s}^{-1}$ (strong storm).

Figure 7 shows $C_{d}$ against wind speed for the weak and strong storms. This can be compared with Fig. 6c, which is the result of the storm with an MWS of 45 $\mathrm{m} \mathrm{s}^{-1}$. These figures show that the dependence of $C_{d}$ on wind speed is very similar except that the decrease of the $C_{d}$ with wind speed in the rear-left quadrant occurs at different wind speeds (at wind speeds just below the MWS). The decrease of $C_{d}$ becomes more distinct as the MWS increases.

\section{c. Effect of storm structure}

When a hurricane moves, actual wind speeds in the right (left) of its track become higher (lower) than those in the left (right) because of addition (subtraction) of the translation speed to the wind speed determined by the hurricane pressure field. The maximum wind speed 


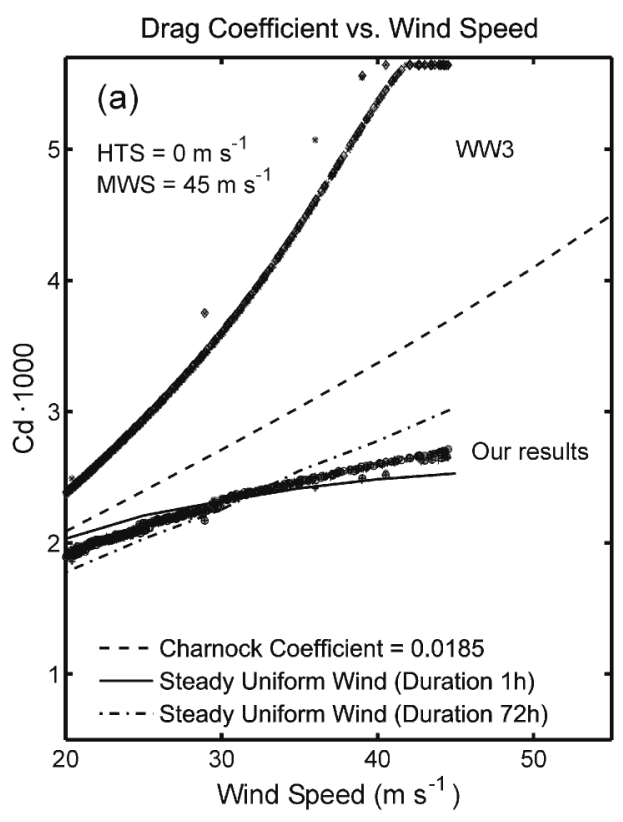

Drag Coefficient vs. Wind Speed

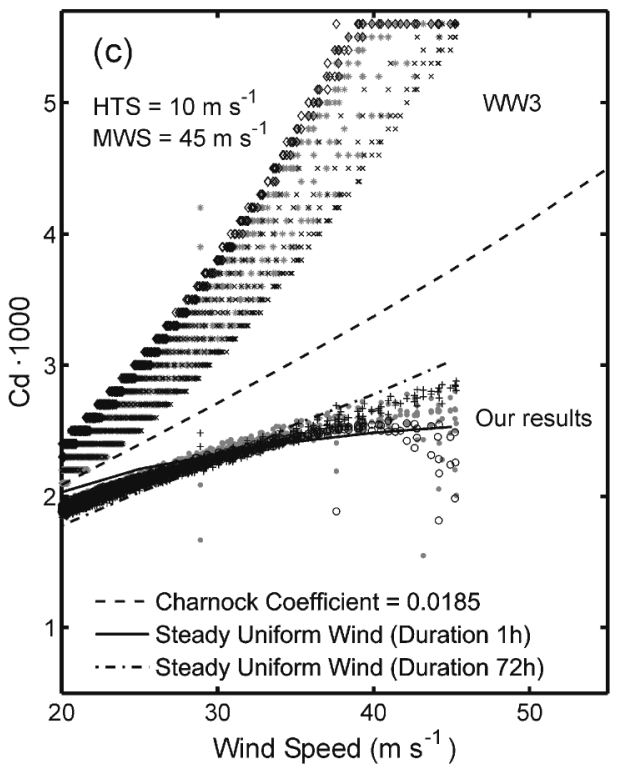

is always found in the right-hand side. In all previous experiments, axisymmetric wind fields (i.e., same wind speeds in all directions from the storm center) are used. Here, we examine the effect of asymmetric wind fields on wind stress, when the hurricane translation speed is added in the applied wind speed.

Figure 8 shows the distribution of the applied wind speed with HTSs of 5 and $10 \mathrm{~m} \mathrm{~s}^{-1}$ and MWS of 45 $\mathrm{m} \mathrm{s}^{-1}$. In Fig. 9 the distributions of waves, wave age, Charnock coefficient, and drag coefficient are shown. These figures can be compared with the symmetric wind field results shown in Fig. 2. The asymmetric effect yields further increase of the wave height, length, and the drag coefficient in the front-right quadrant of the

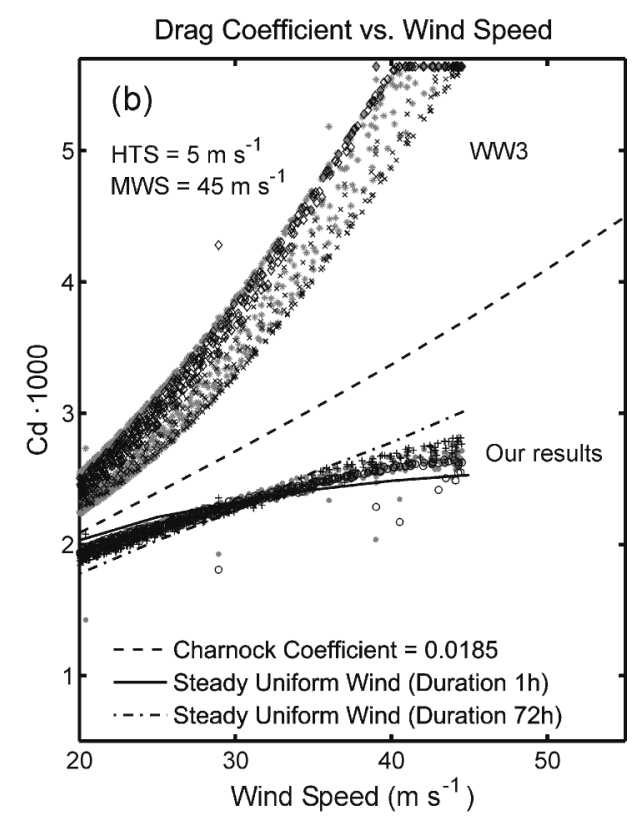

FIG. 6. Scatterplots of the $C_{d}$ as a function of the wind speed in all grid points for storms with translation speeds of (a) 0, (b) 5, and (c) $10 \mathrm{~m} \mathrm{~s}^{-1}$. The solid line and dashed-dotted line represent results of Part I obtained at 1 and $72 \mathrm{~h}$ after the onset of the steady uniform wind, respectively. The dashed line is the bulk formula based on the $z_{\mathrm{ch}}$ of 0.0185 . Our results and WW3's prediction are plotted using different symbols according to the relative position from the storm center (right-front quadrant:,$+ \times$; left-rear quadrant: $\bigcirc, \diamond$; other quadrants: $O, \star)$.

hurricane's track and further decrease of these quantities in the rear-left quadrant. Figure 10 shows $C_{d}$ against wind speed with asymmetric wind forcing. Comparison between Fig. 10 and Figs. 6b and 6c (with symmetric wind forcing) shows that the decrease of the $C_{d}$ for asymmetric wind forcing with wind speed in the rearleft quadrant starts at lower wind speeds compared to the symmetric wind cases.

\section{d. Effect of varying storm speed}

Results presented so far have been obtained after a spinup time of $54 \mathrm{~h}$ with a fixed HTS. This allows us to investigate the behavior of $C_{d}$ under a steady-state 

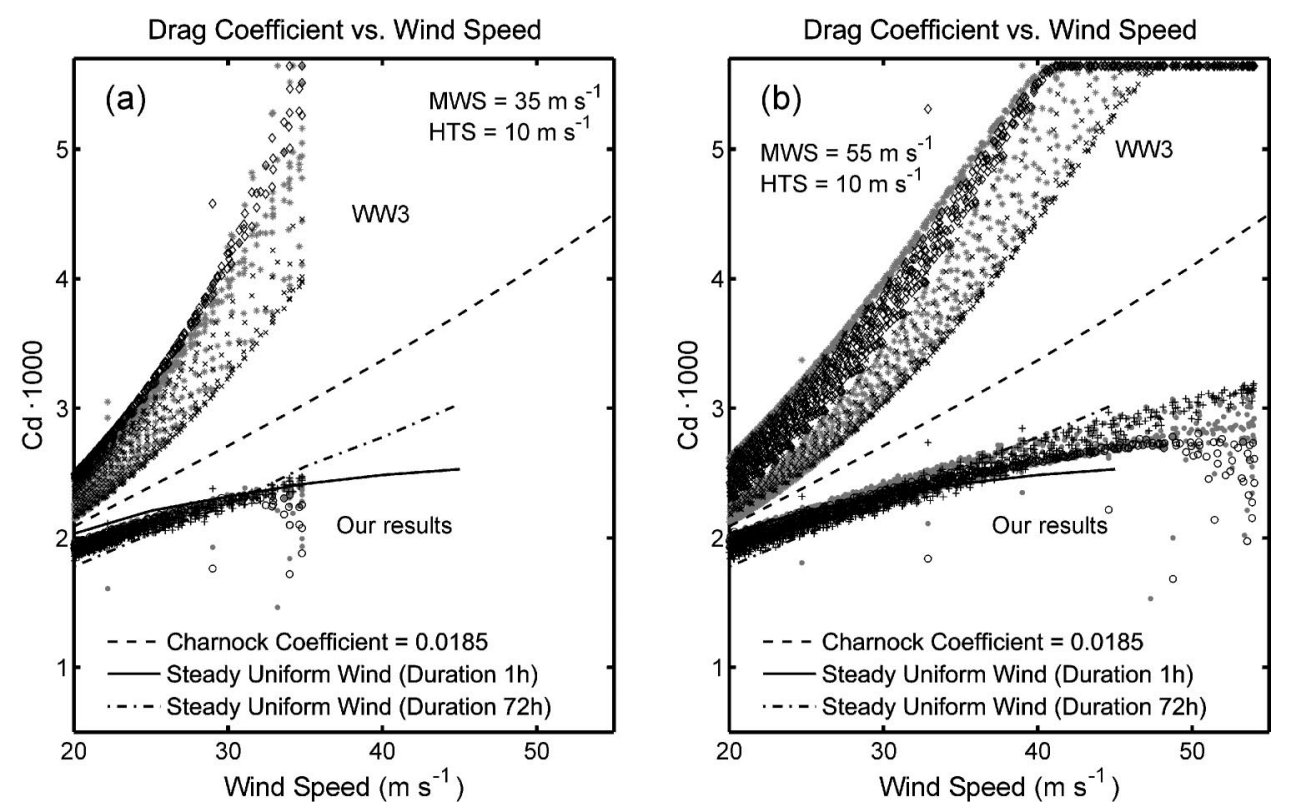

FIG. 7. Same as in Fig. $6 \mathrm{c}$, but for fast-moving (HTS $=10 \mathrm{~m} \mathrm{~s}^{-1}$ ) storms with MWSs of (a) 35 and (b) $55 \mathrm{~m} \mathrm{~s}^{-1}$.

condition (i.e., steady relative to an observer moving with the hurricane). However, as a real hurricane develops, its translation speed often changes significantly. Wind stress fields produced by a varying storm translation speed may differ from the steady-state results with a fixed HTS. In this section, we investigate the effect of a varying HTS on wind stress using the observed translation speed time record of a real hurricane.

Figure 11 shows the translation speed of Hurricane

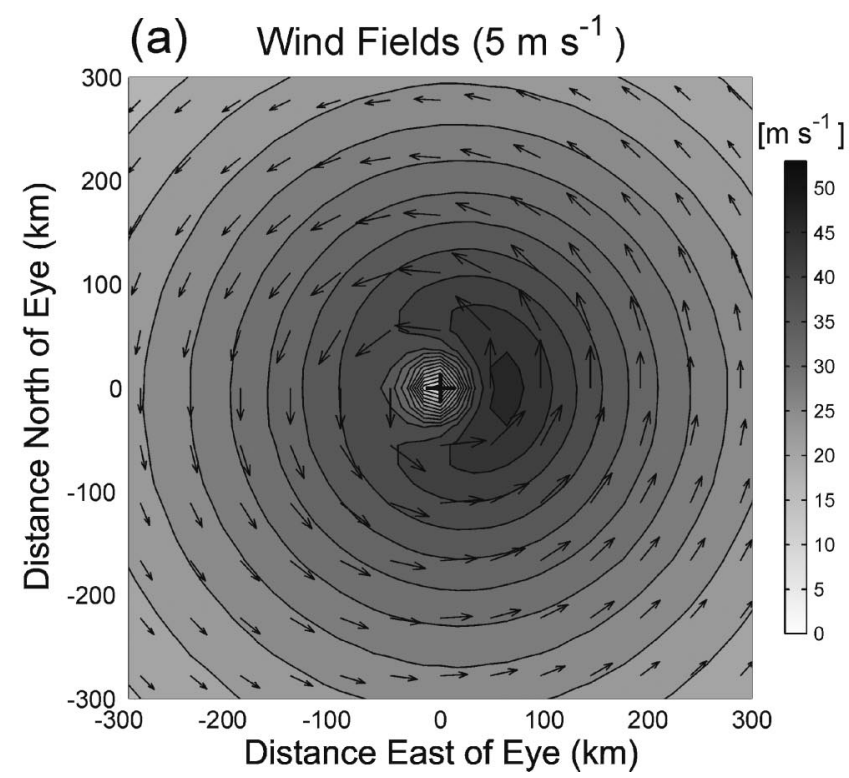

Bonnie (1998) used in this experiment. The speed varies from 2 to $8 \mathrm{~m} \mathrm{~s}^{-1}$ during the 7-day period. For this simulation, an asymmetric wind field, resulting from the summation of the translation speed and an axisymmetric wind field with the MWS of $45 \mathrm{~m} \mathrm{~s}^{-1}$, is applied.

Shown in Fig. 12 are distributions of $C_{d}$ when Bonnie moved at the HTS of $5 \mathrm{~m} \mathrm{~s}^{-1}$ but was accelerating (Fig. 12a, corresponding to point A in Fig. 11) and was decelerating (Fig. 12b, corresponding to point B in Fig.

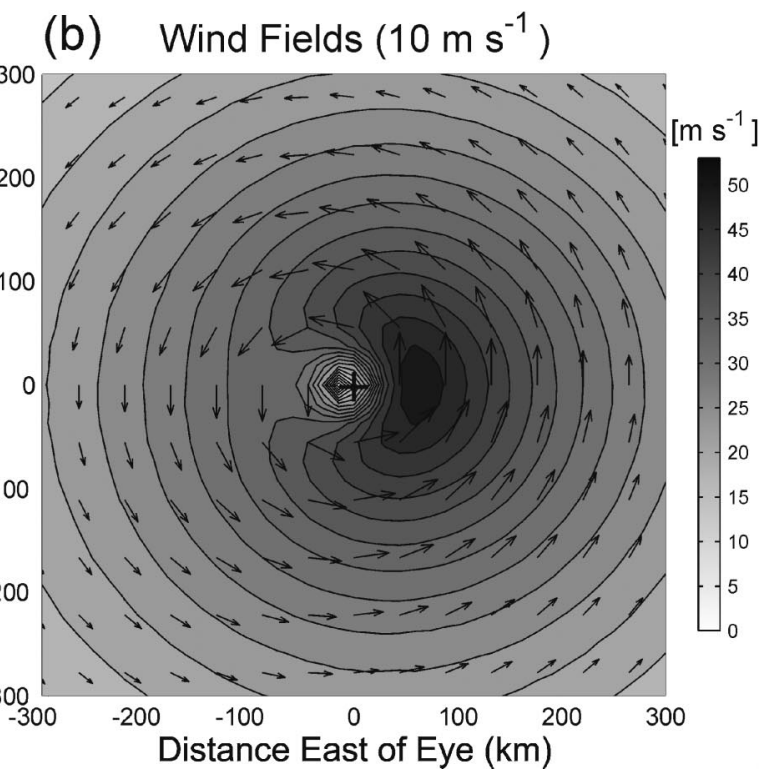

FIG. 8. Spatial distributions of wind produced by asymmetric hurricanes with translation speeds of (a) 5 and (b) $10 \mathrm{~m} \mathrm{~s}^{-1}$. The asymmetric wind fields are calculated from the addition (or subtraction) of the translation speed to wind speed determined by the hurricane pressure field. 
(a) Wave Fields $\left(5 \mathrm{~m} \mathrm{~s}^{-1}\right)$

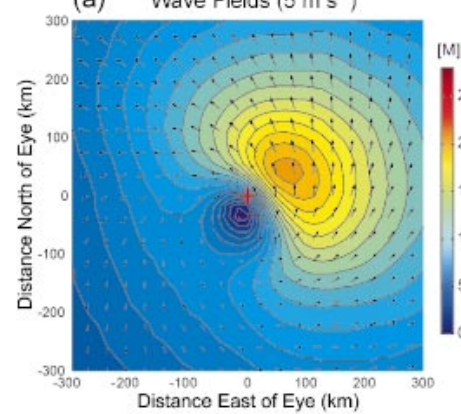

(c) Wave Age $\left(5 \mathrm{~m} \mathrm{~s}^{-1}\right)$

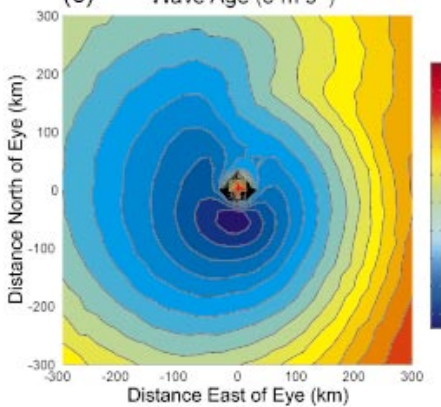

(e) Charnock Coefficient ( $5 \mathrm{~m} \mathrm{~s}^{-1}$ )

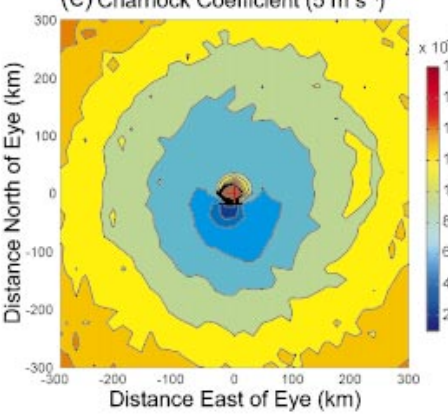

(g) Drag Coefficient (5 m s-1)

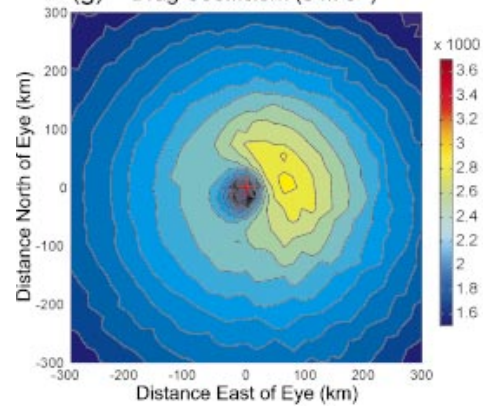

(b) Wave Fields $\left(10 \mathrm{~m} \mathrm{~s}^{-1}\right)$

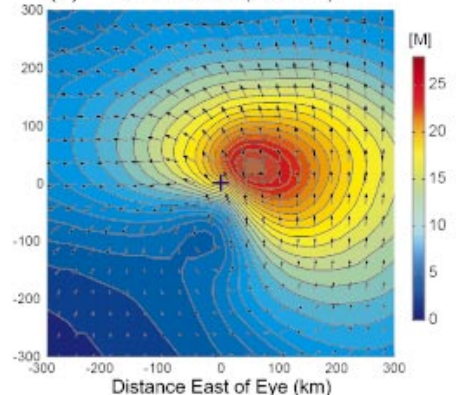

(d) Wave Age $\left(10 \mathrm{~m} \mathrm{~s}^{-1}\right)$

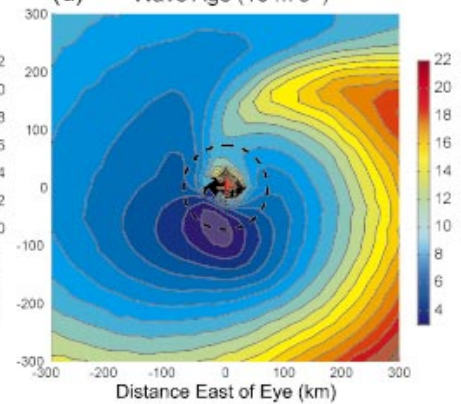

(f) Charnock Coefficient (10 $\mathrm{m} \mathrm{s}^{-1}$ )

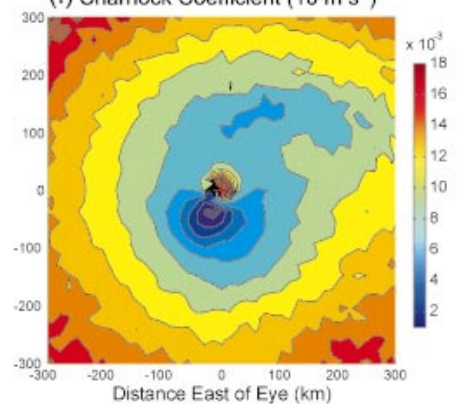

(h) Drag Coefficient $\left(10 \mathrm{~m} \mathrm{~s}^{-1}\right)$

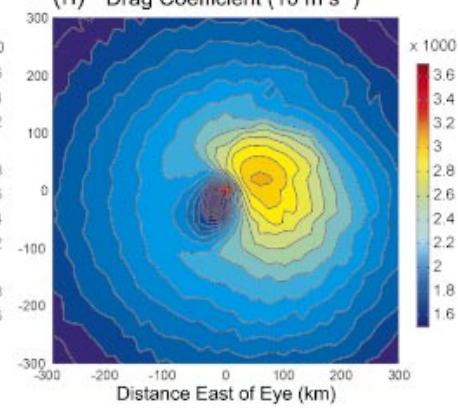

FIG. 9. Same as in Figs. 2b-1, but for asymmetric hurricanes with translation speeds of 5 and $10 \mathrm{~m} \mathrm{~s}^{-1}$.

11). Comparing these figures with Fig. $9 \mathrm{~g}$ (the steadystate result with the same HTS of $5 \mathrm{~m} \mathrm{~s}^{-1}$ ) as well as Fig. $2 \mathrm{j}$ (HTS $=0 \mathrm{~m} \mathrm{~s}^{-1}$ ) and Fig. 9h (HTS $=10 \mathrm{~m}$ $\mathrm{s}^{-1}$ ), one can see that $C_{d}$ during the acceleration is slightly lower than the constant HTS result (Fig. 9g), that is, slightly closer to the HTS $=0 \mathrm{~m} \mathrm{~s}^{-1}$ result (Fig. $2 \mathrm{j}$ ), while $C_{d}$ during the deceleration is higher than the con- stant HTS result (Fig. 9g), that is, closer to the HTS = $10 \mathrm{~m} \mathrm{~s}^{-1}$ result (Fig. 9h). The same trend is also found in the scatterplots of $C_{d}$ against the wind speed (not shown here). This is mainly because the surface wave field is not determined by the instantaneous wind forcing but by the time history of wind forcing; that is, the wave field retains the memory of the wind-forcing history. 

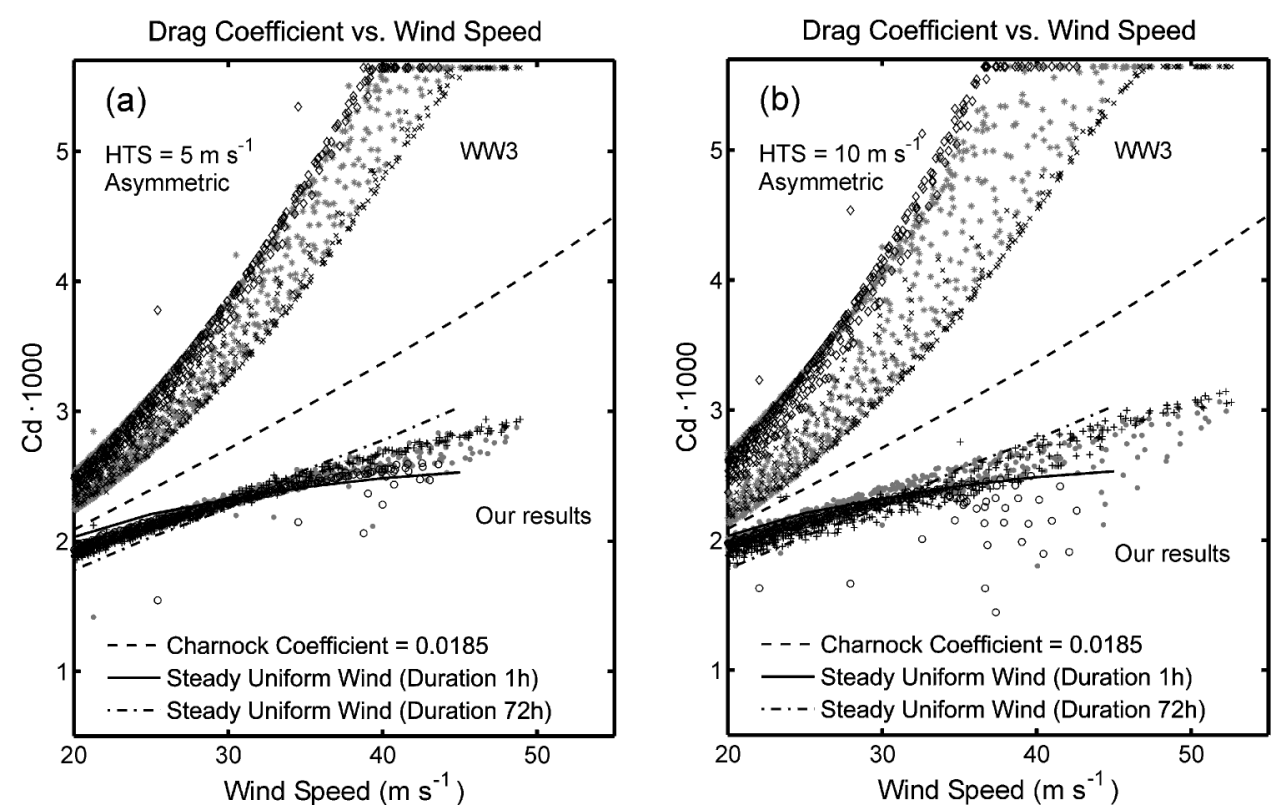

FIG. 10. Same as in Figs. $6 \mathrm{~b}$ and $6 \mathrm{c}$, but for asymmetric hurricanes with translation speeds of 5 and $10 \mathrm{~m} \mathrm{~s}^{-1}$.

Consequently, the drag coefficient, which is influenced by the surface wave field, responds to varying wind forcing with some time delay.

\section{e. Comparison with other studies}

Figure 13 compares our results of $C_{d}$ against wind speed with two existing parameterizations [the formula by Large and Pond (1981) and the bulk formula with a constant Charnock coefficient of 0.0185], the WW3 prediction, and the estimate based on observations of Powell et al. (2003). The latter is the only estimate reported under hurricane forcing of wind speed over $40 \mathrm{~m} \mathrm{~s}^{-1}$. Our upper and lower bounds are determined by combining the $C_{d}$ values from all experiments described

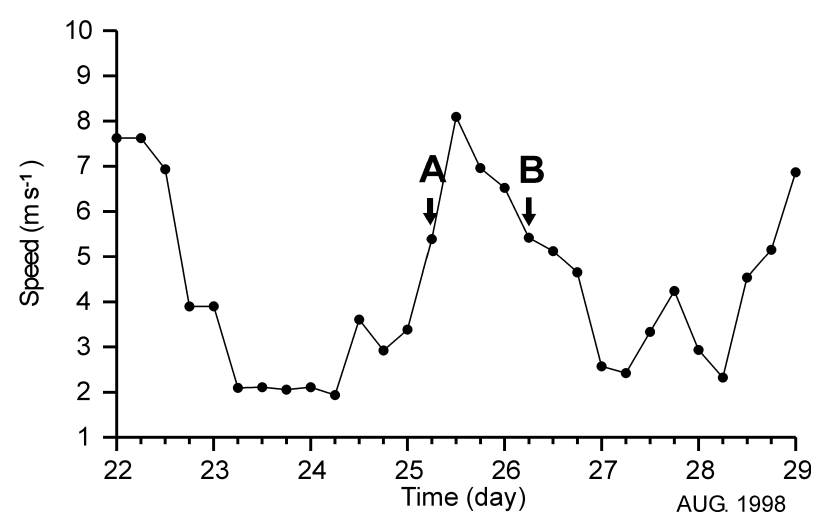

FIG. 11. Variation of Hurricane Bonnie translation speed. Two points (A and B) with an HTS of $5 \mathrm{~m} \mathrm{~s}^{-1}$ but accelerating or decelerating speed are chosen to compare with the steady-state results with the same speed. earlier. Most data points are located near the upper bound. Data points near the lower bound are from the rear-left quadrant of the hurricane.

At high wind speeds, our overall tendency for $C_{d}$ to level off and even decrease with wind speed is significantly different from that of the two surface flux parameterizations that are widely used in a variety of models, including those used for forecasting hurricanes and storm surges. The existing parameterizations yield a monotonic increase of $C_{d}$ with wind speed because they are based on extrapolations from the measurements obtained at lower winds. The internal prediction of the WW3 based on the sea-state-dependent $C_{d}$ shows an even faster increase at high wind speeds, because it assumes that younger waves yield higher drag regardless of wind speeds, while our model predicts lower drag with younger waves at high wind speeds.

Powell et al. (2003) estimated $C_{d}$ using a dataset from hundreds of global positioning system (GPS) sondes that were dropped in the vicinity of hurricane eyewalls, where the strongest wind occurs, in the Atlantic basin and in the eastern and central Pacific basins since 1997. This is among the first estimates of $C_{d}$ in tropical cyclones under high wind speeds over $40 \mathrm{~m} \mathrm{~s}^{-1}$. Figure 13 shows that for high wind speeds above $35 \mathrm{~m} \mathrm{~s}^{-1}$, their estimation is much less than the two existing parameterizations. The overall trend of the estimated $C_{d}-$ that is, increasing with wind speeds at lower wind speeds and decreasing with wind speeds at very higher winds-is qualitatively consistent with our model results.

A leveling off and decrease of $C_{d}$ at high wind speeds was also found in laboratory experiments by Alamaro 
(a) Drag Coefficient (06h 25 AUG)

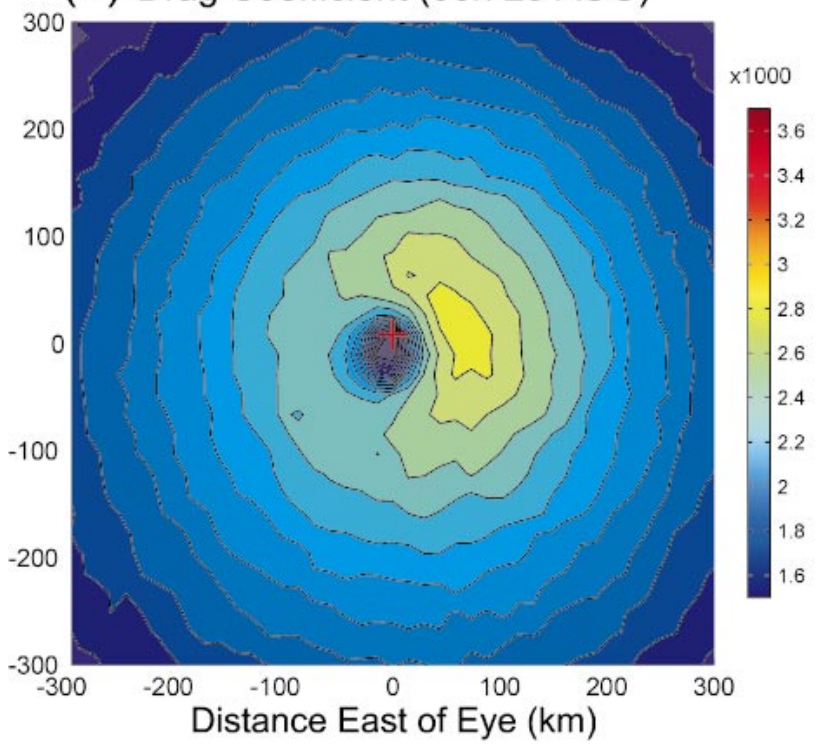

(b) Drag Coefficient (06h 26 AUG)

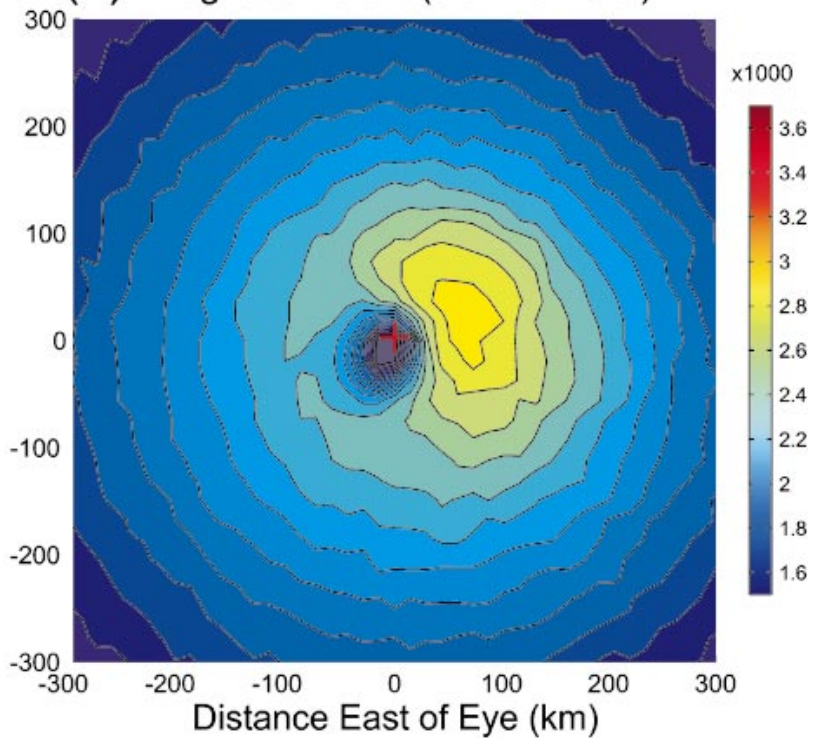

FIG. 12. Spatial distributions of $C_{d}$ produced by an idealized storm using the translation speeds of Hurricane Bonnie at (a) 0600 UTC 25 Aug and (b) 0600 UTC 26 Aug 1998, which correspond with the times identified as points A and B in Fig. 11.

et al. (2002). In addition, Emanuel (2003) predicts that the drag coefficients should be capped at a constant value at sufficiently high wind speeds based on his theoretical study of the energy balance of a hurricane system. Our model results are consistent with these recent studies as well. Moreover, our model results provide a plausible explanation as to why the drag coefficient ceases to increase at very high wind speeds.

\section{Summary and conclusions}

The effect of surface waves on air-sea momentum exchange under hurricane wind forcing has been investigated by combining the WAVEWATCH-III ocean wave model, the spectral tail parameterization based on the equilibrium spectral model by Hara and Belcher (2002), and the wave boundary layer model of Hara and Belcher (2004). The combined model estimates the wind stress vector by explicitly calculating the wave-induced stress vector. This approach allows us to estimate the drag coefficient and the equivalent surface roughness for any surface wave fields, even for complex seas driven by hurricanes. From the numerical experiments using idealized hurricanes with different translation speeds, intensities, and structures, we have investigated the behavior of the drag coefficient $C_{d}$ and the Charnock coefficient $z_{\mathrm{ch}}$ under hurricane wind forcing. The results can be summarized as follows.

1) Under hurricane wind forcing, dominant waves often propagate with a large angle to the local wind. However, the misalignment between the wind stress direction and the wind direction is always small (less than a few degrees). This is mainly because under strong wind forcing, a significant amount of wind stress is supported by wind-forced waves that are aligned with local wind. Consequently, the longer waves that are misaligned with local wind support little momentum flux; they may affect the wind stress magnitude but may not affect the wind stress direction significantly. Therefore, it is possible to define and calculate the drag coefficient and the equivalent surface roughness using the wind stress magnitude and the wind speed magnitude.

2) As the hurricane translation speed increases, higher, longer, and more developed waves in the right-front quadrant of the storm track produce higher sea drag; lower, shorter, and younger waves in the rear-left quadrant produce lower sea drag.

3) The Charnock coefficient $z_{\text {ch }}$ is mainly determined by the local wind speed and the local input wave age (wave age of local wind seas) regardless of the complexity of the wave field. For lower wind speeds, $z_{\mathrm{ch}}$ tends to decrease with the input wave age; for strong winds above $30 \mathrm{~m} \mathrm{~s}^{-1}, z_{\mathrm{ch}}$ tends to decrease as waves become younger. The latter trend is different from those found in most previous observational and theoretical studies because such a trend is found only in extreme young seas and high winds.

4) The drag coefficient increases with wind speed at lower winds, but the rate of its increase is significantly reduced at high winds and there is a leveling off or even decrease in certain sectors. This is because young waves produced in high winds (especially in the rear-left quadrant of the storm track) generate small drags. The spatial variability of the drag coefficient increases as the hurricane translation speed increases. 


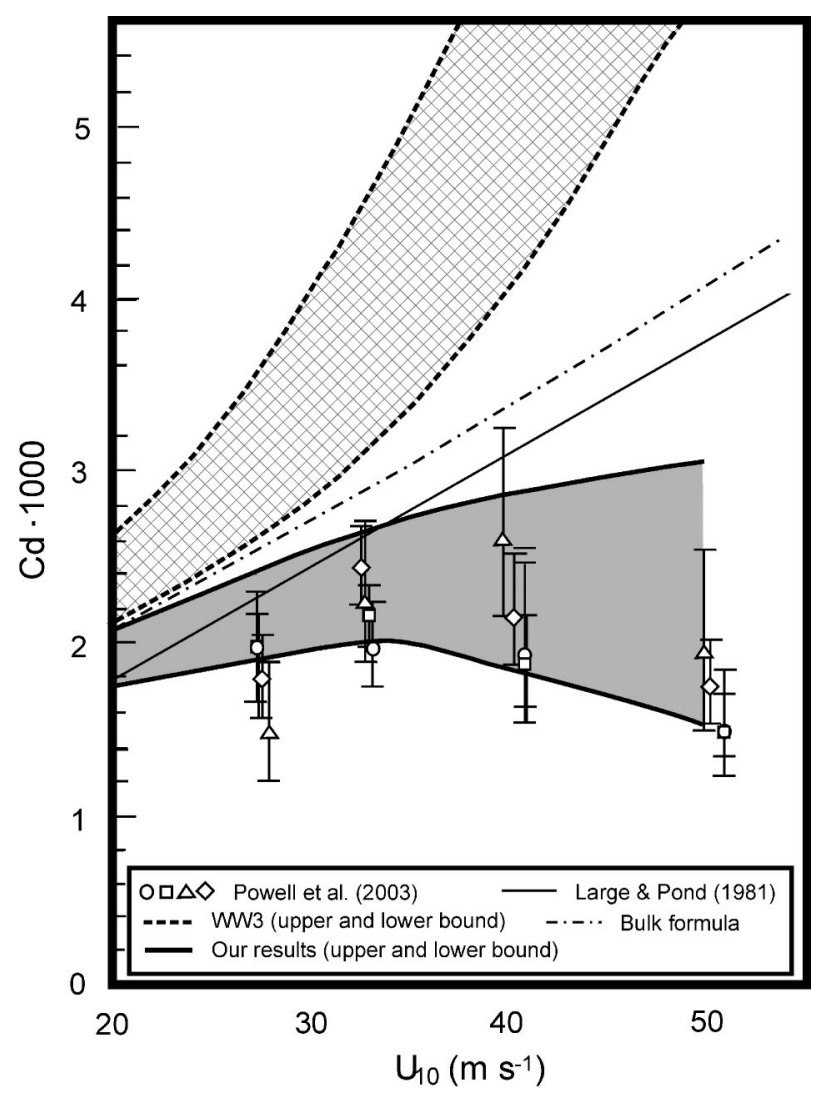

FIG. 13. Comparison of $C_{d}$ between various observation-based values (or formulas) and model outputs as a function of wind speed. Symbols represent $C_{d}$ estimated from GPS sonde data in various hurricanes (Powell et al. 2003). Vertical bars represent the range of estimates based on $95 \%$ confidence. The solid line is an extrapolation of the formula by Large and Pond (1981). The dashed-dotted line is the bulk formula based on the $z_{\mathrm{ch}}$ of 0.0185 . Shaded and stippled areas represent ranges between upper and lower bounds of $C_{d}$ obtained by the present approach and the WW3 for all experiment cases, respectively.

5) Asymmetric wind forcing (resulting from adding the hurricane translation speed to an axisymmetric wind speed) contributes to further increase of the wave height, length, and sea drag in the front-right quadrant of the storm track and further decrease of these in the rear-left quadrant.

6) When the hurricane translation speed changes, $C_{d}$ responds with some time delay because the surface wave field retains a memory of the wind-forcing history.

7) Our numerical experiments with different hurricane conditions generally predict an overall tendency that $C_{d}$ increases with wind speed at low winds and levels off with wind speed at very high winds. This tendency is consistent with the recent laboratory study by Alamaro et al. (2002) and the theoretical study by Emanuel (2003). An evenly reduced $C_{d}$ with wind speed, which is found in certain sectors of very high wind regimes, is also consistent with the result of
Powell et al. (2003) obtained from various tropical cyclones. The existing parameterizations and the WW3 internal calculations significantly overestimate the drag coefficient at high winds.

An important conclusion that follows from this study is that accurate hurricane forecasting requires coupling between a hurricane model and a surface wave model, since accurate prediction of surface fluxes are crucial for hurricane forecasting, in particular, for storm intensity and track prediction. Experiments with the GFDL/ University of Rhode Island (URI) coupled hurricaneocean model indicate important sensitivity of the hurricane intensity to the momentum flux parameterization at the sea surface (Ginis et al. 2004). In these experiments, if the roughness length is set constant (capped) for wind speeds greater than $35 \mathrm{~m} \mathrm{~s}^{-1}$, consistent with our results, it leads to a substantial increase of maximum surface wind. The changes of the wind speed extend through the boundary layer. These results strongly suggest that the hurricane intensity predictions (as well as corresponding track forecast) could be significantly affected by the improved parameterization of the air-sea momentum flux at high wind speeds.

Hurricane winds produce a large number of breaking waves and sea spray. Breaking waves and spray may significantly change the wind stress. Makin and $\mathrm{Ku}-$ dryavtsev (2002) predicted that breaking waves significantly enhance the sea drag over younger seas. Andreas and Emanuel (2001) considered spray effects on momentum transfer and concluded that this effect could be large as well. It is our intention to include these processes in our future modeling efforts. It is important to realize, however, that estimating quantitatively the effect of breaking waves and spray is very difficult because our quantitative understanding of such processes is extremely limited at present. The modeling study presented here, although it neglects breaking waves and spray, provides a plausible and quantitative explanation as to why the drag coefficient under tropical cyclones is significantly reduced at high wind speeds.

Acknowledgments. This work was supported by the U.S. National Science Foundation (Grant ATM 0001038). TH also thanks the U.S. Office of Naval Research (CBLAST program, Grant N00014-0110125) for additional support.

\section{REFERENCES}

Alamaro, M., K. Emanuel, J. Colton, W. McGillis, and J. B. Edson, 2002: Experimental investigation of air-sea transfer of momentum and enthalpy at high wind speed. Preprints, 25th Conf. on Hurricanes and Tropical Meteorology, San Diego, CA, Amer. Meteor. Soc., 667-668.

Andreas, E. L, and K. Emanuel, 2001: Effect of sea spray on tropical cyclone intensity. J. Atmos. Sci., 58, 3741-3751.

Bao, J.-W., J. M. Wilczak, J.-K. Choi, and L. H. Kantha, 2000: Numerical simulations of air-sea interaction under high wind con- 
ditions using a coupled model: A study of hurricane development. Mon. Wea. Rev., 128, 2190-2210.

Bourassa, M. A., D. G. Vincent, and W. L. Wood, 1999: A flux parameterization including the effects of capillary waves and sea state. J. Atmos. Sci., 56, 1123-1139.

Chalikov, D. V., and V. K. Makin, 1991: Models of the wave boundary layer. Bound.-Layer Meteor., 56, 83-99.

Charnock, H., 1955: Wind stress on a water surface. Quart. J. Roy. Meteor. Soc., 81, 639-640.

Desjardins, S., J. Mailhot, and R. Lalbeharry, 2000: Examination of the impact of a coupled atmospheric and ocean wave system. Part I: Atmospheric aspects. J. Phys. Oceanogr., 30, 385-401.

Doyle, J. D., 2002: Coupled atmosphere-ocean wave simulations under high wind conditions. Mon. Wea. Rev., 130, 3087-3099.

Drennan, W. M., G. C. Graber, D. Hauser, and C. Quentin, 2003: On the wave age dependence of wind stress over pure wind seas. J. Geophys. Res., 108, 8062, doi:10.1029/2000JC000715.

Emanuel, K. A., 1995: Sensitivity of tropical cyclones to surface exchange coefficients and a revised steady-state model incorporating eye dynamics. J. Atmos. Sci., 52, 3969-3976.

— 2003 : A similarity hypothesis for air-sea exchange at extreme wind speeds. J. Atmos. Sci., 60, 1420-1428.

Geernaert, G. L., 1988: Measurements of the angle between the wind vector and wind stress vector in the surface layer over the North Sea. J. Geophys. Res., 93, 8215-8220.

Ginis, I., I.-J. Moon, B. Thomas, and T. Hara, 2004: Effect of ocean waves on air-sea momentum fluxes and hurricane intensity. Preprints, 26th Conf. on Hurricanes and Tropical Meteorology, Miami, FL, Amer. Meteor. Soc., 34-35.

Grachev, A. A., C. W. Fairall, J. E. Hare, J. B. Edson, and S. D. Miller, 2003: Wind stress vector over ocean waves. J. Phys. Oceanogr., 33, 2408-2429.

Hara, T., and S. E. Belcher, 2002: Wind forcing in the equilibrium range of wind-wave spectra. J. Fluid Mech., 470, 223-245.

— ocean surface wave spectra. J. Phys. Oceanogr., in press.

Holland, G. J., 1980: An analytic model of the wind and pressure profiles in hurricanes. Mon. Wea. Rev., 108, 1212-1218.

Janssen, P. A. E. M., 1989: Wave-induced stress and the drag of air flow over sea waves. J. Phys. Oceanogr., 19, 745-754.

Johnson, H. K., J. Hfjstrup, H. J. Vested, and S. E. Larsen, 1998: On the dependence of sea surface roughness on wind waves. J. Phys. Oceanogr., 28, 1702-1716.

-, H. J. Vested, H. Hersbach, J. Hojstrup, and S. E. Larsen, 1999: The coupling between wind and waves in the WAM model. $J$. Atmos. Oceanic Technol., 16, 1780-1790.

Jones, I. S. F., and Y. Toba, 2001: Wind Stress over the Ocean. Cambridge University Press, $303 \mathrm{pp}$.

Komen, G. J., P. A. E. M. Janssen, V. Makin, and W. Oost, 1998: On the sea state dependence of the Charnock parameter. Global Atmos. Ocean Syst., 5, 367-388.

Kurihara, Y., R. E. Tuleya, and M. A. Bender, 1998: The GFDL hurricane prediction system and its performance in the 1995 hurricane season. Mon. Wea. Rev., 126, 1306-1322.

Large, W. G., and S. Pond, 1981: Open ocean momentum flux mea- surements in moderate to strong wind. J. Phys. Oceanogr., 11, 324-336.

Lionello, P., P. Malguzzi, and A. Buzzi, 1998: Coupling between the atmospheric circulation and the ocean wave field: An idealized case. J. Phys. Oceanogr., 28, 161-177.

Makin, V. K., and C. Mastenbroek, 1996: Impact of waves on airsea exchange of sensible heat and momentum. Bound.-Layer Meteor., 79, 279-300.

_ , and V. N. Kudryavtsev, 1999: Coupled sea surface-atmosphere model. 1. Wind over waves coupling. J. Geophys. Res., 104, 7613-7623.

- and - 2002: Impact of dominant waves on sea drag. Bound. Layer Meteor., 103, 83-99.

_, _ _ a and C. Mastenbroek, 1995: Drag of the sea surface. Bound.-Layer Meteor., 73, 159-182.

Monin, A. S., and A. M. Obukhov, 1954: Basic laws of turbulent mixing in the surface layer of the atmosphere. Tr. Geofiz. Inst., Akad. Navk SSSR, 24, 163-187.

Moon I.-J, 2004: Impact of a coupled ocean wave-tide-circulation system on coastal modeling. Ocean Modell., in press.

_ - I. Ginis, T. Hara, H. Tolman, C. W. Wright, and E. J. Walsh, 2003a: Numerical simulation of sea surface directional wave spectra under hurricane wind forcing. J. Phys. Oceanogr., 33, 1680-1706.

- I. S. Oh, T. Murty, and Y. H. Yoon, 2003b: Causes of the unusual coastal flooding generated by Typhoon Winnie on the west coast of Korea. Nat. Hazards, 29, 485-500.

- T. Tara, I. Ginis, S. E. Belcher, and H. Tolman, 2004: Effect of surface waves on air-sea momentum exchange. Part I: Effect of mature and growing seas. J. Atmos. Sci., 61, 2321-2333.

Ooyama, K., 1969: Numerical simulation of the life cycle of tropical cyclones. J. Atmos. Sci., 26, 3-40.

Powell, M. D., P. J. Vickery, and T. A. Reinhold, 2003: Reduced drag coefficient for high wind speeds in tropical cyclones. Nature, 422, 279-283.

Rieder, K. F., J. A. Smith, and R. A. Weller, 1994: Observed directional characteristics of the wind, wind stress and surface waves on the open ocean. J. Geophys. Res., 99, 22 596-22 598.

Smith, S. D., 1980: Wind stress and heat flux over the ocean in gale force winds. J. Phys. Oceanogr., 10, 709-726.

— ficients: The HEXOS results. Bound.-Layer Meteor., 60, 109142.

Toba, Y., N. Iida, H. Kawamura, N. Ebuchi, and I. S. F. Jones, 1990: The wave dependence of sea-surface wind stress. J. Phys. Oceanogr., 20, 705-721.

Tolman, H. L., 2002: User manual and system documentation of WAVEWATCH-III version 2.22. NOAA/NWS/NCEP/OMB Tech. Note 222, 133 pp.

— wave model. J. Phys. Oceanogr., 26, 2497-2518.

WAMDI Group, 1988: The WAM model-A third generation ocean wave prediction model. J. Phys. Oceanogr., 18, 1775-1810.

Weber, S., H. von Storch, P. Viterbo, and L. Zambresky, 1993: Coupling an ocean wave to an atmospheric general circulation model. Climate Dyn., 9, 63-69. 